\title{
The cosmic ray energy spectrum measured with the new Tibet hybrid experi- ment
}

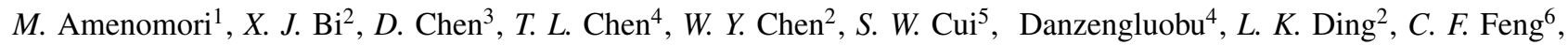

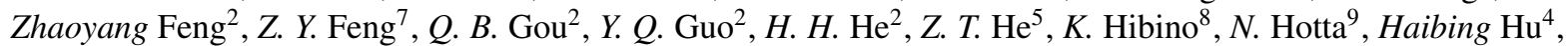
H. B. $\mathrm{Hu}^{2}, J$. Huang $^{2, *}, H . Y . \mathrm{Jia}^{7}, L . \mathrm{Jiang}^{2}, F . \mathrm{Kajino}^{10}, K . \mathrm{Kasahara}^{11}, Y$. Katayose ${ }^{12}, C . \mathrm{Kato}^{13}, K . \mathrm{Kawata}^{14}, M$. Kozai $^{13,15}$, Labaciren ${ }^{4}$, G. M. Le ${ }^{16}, A . F . \mathrm{Li}^{17,6,2}, H . J . \mathrm{Li}^{4}$, W. J. Li ${ }^{2,7}, Y . H . \mathrm{Lin}^{2,18}, C . \mathrm{Liu}^{2}, J . S . \mathrm{Liu}^{2}, M . Y . \mathrm{Liu}^{4}, H . \mathrm{Lu}^{2}$, $X . R$. Meng $^{4}, T$. Miyazaki $^{13}, K$. Munakata $^{13}, T$. Nakajima $^{13}, Y$. Nakamura $^{13}, H$. Nanjo $^{1}, M$. Nishizawa $^{19}, T$. Niwa $^{13}$,

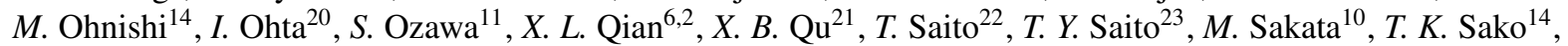

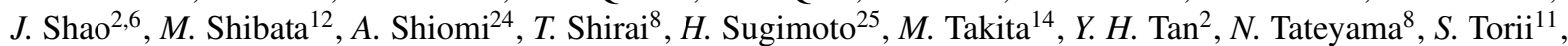
$H$. Tsuchiya ${ }^{26}, S . \mathrm{Udo}^{8}, H$. Wang ${ }^{2}, H . R . \mathrm{Wu}^{2}, L . \mathrm{Xue}^{6}, Y$. Yamamoto ${ }^{10}, K$. Yamauchi ${ }^{12}, Z$. Yang ${ }^{2}, A . F$. Yuan $^{4}, L . M$.

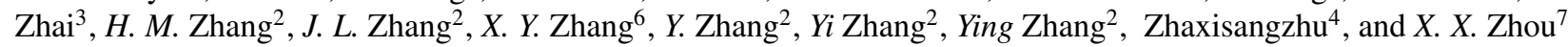
(The Tibet AS $\gamma$ Collaboration)

${ }^{1}$ Department of Physics, Hirosaki University, Hirosaki 036-8561, Japan

${ }^{2}$ Key Laboratory of Particle Astrophysics, Institute of High Energy Physics, Chinese Academy of Sciences, Beijing 100049, China

${ }^{3}$ National Astronomical Observatories, Chinese Academy of Sciences, Beijing 100012, China

${ }^{4}$ Department of Mathematics and Physics, Tibet University, Lhasa 850000, China

${ }^{5}$ Department of Physics, Hebei Normal University, Shijiazhuang 050016, China

${ }^{6}$ Department of Physics, Shandong University, Jinan 250100, China

${ }^{7}$ Institute of Modern Physics, SouthWest Jiaotong University, Chengdu 610031, China

${ }^{8}$ Faculty of Engineering, Kanagawa University, Yokohama 221-8686, Japan

${ }^{9}$ Faculty of Education, Utsunomiya University, Utsunomiya 321-8505, Japan

${ }^{10}$ Department of Physics, Konan University, Kobe 658-8501, Japan

${ }^{11}$ Research Institute for Science and Engineering, Waseda University, Tokyo 169-8555, Japan

${ }^{12}$ Faculty of Engineering, Yokohama National University, Yokohama 240-8501, Japan

${ }^{13}$ Department of Physics, Shinshu University, Matsumoto 390-8621, Japan

${ }^{14}$ Institute for Cosmic Ray Research, University of Tokyo, Kashiwa 277-8582, Japan

${ }^{15}$ Institute of Space and Astronautical Science, Japan Aerospace Exploration Agency (ISAS/JAXA), Sagamihara 252-5210, Japan

${ }^{16}$ National Center for Space Weather, China Meteorological Administration, Beijing 100081, China

${ }^{17}$ School of Information Science and Engineering, Shandong Agriculture University, Taian 271018, China

${ }^{18}$ University of Chinese Academy of Sciences, Beijing 100049, China

${ }^{19}$ National Institute of Informatics, Tokyo 101-8430, Japan

${ }^{20}$ Sakushin Gakuin University, Utsunomiya 321-3295, Japan

${ }^{21}$ College of Science, China University of Petroleum, Qingdao 266555, China

${ }^{22}$ Tokyo Metropolitan College of Industrial Technology, Tokyo 116-8523, Japan

${ }^{23}$ Max-Planck-Institut für Physik, Munich D-80805, Germany

${ }^{24}$ College of Industrial Technology, Nihon University, Narashino 275-8576, Japan

${ }^{25}$ Shonan Institute of Technology, Fujisawa 251-8511, Japan

${ }^{26}$ Japan Atomic Energy Agency, Tokai-mura 319-1195, Japan

\begin{abstract}
We have upgraded the new Tibet ASgamma experiment in China since 2014 to measure the chemical composition of cosmic rays around the knee. This hybrid experiment consist of an air-shower-core detector array (YAC-II) to detect high energy electromagnetic component, the Tibet air-shower array (Tibet-III) and a large underground water-Cherenkov muon-detector array (MD). We have carried out a detailed air-shower Monte Carlo (MC) simulation to study the performance of the hybrid detectors by using CORSIKA (version 7.5000), which includes EPOS-LHC, QGSJETII-04, SIBYLL2.1 and SIBYLL2.3 hadronic interaction models. The preliminary results of the interaction model checking above $50 \mathrm{TeV}$ energy region are reported in this paper, and the primary proton and helium spectra in the energy range $50 \mathrm{TeV}$ to $10^{15} \mathrm{eV}$ was derived from YAC-I data and is smoothly connected with direct observation data at lower energies and also with our previously reported works at higher energies within statistical errors. The knee of the $(\mathrm{P}+\mathrm{He})$ spectra is located around $400 \mathrm{TeV}$. The interaction model dependence in deriving the primary $(\mathrm{P}+\mathrm{He})$ spectra is found to be small (less than $25 \%$ in absolute intensity, $10 \%$ in position of the knee), and the composition model dependence is less than $10 \%$ in absolute intensity.
\end{abstract}




\section{Introduction}

The all-particle energy spectrum of primary cosmic rays can be well described by a power law $\mathrm{dj} / \mathrm{dE} \propto \mathrm{E}^{-\gamma}$ over many orders of magnitude, with the power index $\gamma$ changing sharply from 2.7 to 3.1 at about $4 \mathrm{PeV}[1,2]$. Such break structure of the all-particle energy spectrum is called the "knee", and the corresponding energy range is the so called "knee region". The special structure of the power law spectrum is considered to be closely related to the origin, acceleration and propagation mechanism of cosmic rays in the galaxy; however, its origin has not been well understood [3] due to the lack of detailed information about the chemical composition around the knee. The best ways to study the chemical compositions are direct measurements of primary cosmic rays by balloon flights or satellites, but the energy range with sufficient event statistics is limited to $10^{14} \mathrm{eV}$ because of limited exposure time and small detective area. So the task of studying chemical components of knee region still relies on ground-based indirect measurements. The early works doing the research of the chemical composition of the knee region were the Tibet emulsion chamber (Tibet-EC) experiment [4] and the KASCADE experiment [5]. The results of the Tibet-EC suggest that the main component responsible for making the knee structure is composed of nuclei heavier than helium, however, the KASCADE claims that the knee is due to the steepening of the spectra of light elements with an exponential type cutoff as shown in Fig.1. It is also noted that the experimental data points are still poor in the energy range between the direct measurements and the indirect measurements waiting for us to study. Explicit determination of the break point of the spectral index for individual chemical component is essentially important for the study of the cosmic-ray origin.

In order to explicitly observe the break point of the spectral index for individual chemical component, we have recently upgraded the Tibet-EC experiment and started a new Tibet-YAC experiment, for reasons as follow: The Tibet-EC experiment was carried out to measure the Proton and Helium spectrum around the knee by detecting high energy electromagnetic particles at the air-shower core with a detection threshold of the core energy $20 \mathrm{TeV}$ (the corresponding primary energy is several times 100 $\mathrm{TeV})$. Such high-energy shower cores can be mostly generated by light primary nuclei penetrating deep in the atmosphere. It could reveal the energy spectrum of Proton and Helium in the energy range $5 \times 10^{14} \mathrm{eV}-10^{16} \mathrm{eV}$ covering the knee. The result showed steep power indices of Proton and Helium close to 3.1, which is apparently larger than the results by direct observations (about 2.7) measured below $10^{14} \mathrm{eV}$. The break points of the power indices of the Proton and Helium spectra are expected at an energy between $10^{14} \mathrm{eV}$ and $10^{15} \mathrm{eV}$, however there are still poor experimental data in this energy range. The extension of the Tibet-EC experiment for a wider energy range needed a new AS core detector with lower detection threshold energy and wider dynamic range with good geometrical uniformity for the incident particles upon a detector. The YAC

*e-mail: huangjing@ihep.ac.cn

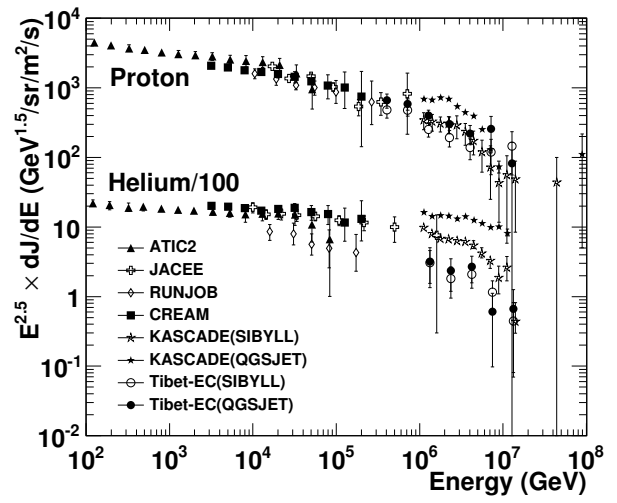

Figure 1. Energy spectra of primary cosmic-ray proton and helium nuclei obtained by Tibet-EC [4] compared with some other experimental results: ATIC2 [6], JACEE [7], RUNJOB [8], CREAM [9], KASCADE [5].

detector was developed to satisfy these demands. The detection threshold energy of the YAC can be set at several 10 times lower than $\mathrm{EC}(300 \mathrm{GeV}$, corresponding primary energy is several times $10 \mathrm{TeV}$ ) by adopting the scintillator instead of the X-ray film for the detection of cascade showers induced in the lead plate by high energy electromagnetic particles at the AS core, and the wide dynamic range of $1-10^{6}$ MIPs (Minimum Ionization Particles) for the burst size detection is realized by installing 2 PMTs (high gain PMT and low gain PMT). Use of the wave length shifting fiber to collect the scintillating light improves the geometrical uniformity. This new experimental condition improves the statistics of the high energy core event compared with the Tibet-EC experiment by a factor of 100. The new hybrid experiment (YAC+Tibet-III+MD) aims to observe the energy spectrum of Proton and Helium whose energy range will overlap with direct observations at lower energies such as CREAM, ATIC and TRACER, and Tibet-EC experiment at higher energies. On the other hand, we added underground muon detector to the new Tibet hybrid experiment, to measure the muon component of the cosmic rays at the high attitude where dependence on hadronic interaction models is expected to be much less than the case of sea-level observations. So the new Tibet hybrid experiment (YAC+Tibet-III+MD) consists of the Yangbajing air-shower Core detector array (YAC), the Tibet air-shower array (Tibet-III) and an underground waterCherenkov muon-detector array (MD). In this paper, we will report the primary proton and helium spectra in the energy range from $50 \mathrm{TeV}$ to $10^{15} \mathrm{eV}$ derived from YAC-I data based on the newest interaction models (EPOS-LHC, QGSJETII-04, SIBYLL2.1 and SIBYLL2.3).

\section{Experiment}

Aiming at the observation of cosmic-ray chemical composition in the knee energy region, a new type air-showercore detector (YAC, Yangbajing Air shower Core array) has been developed and set up at Yangbajing, $4300 \mathrm{~m}$ a.s.l. 


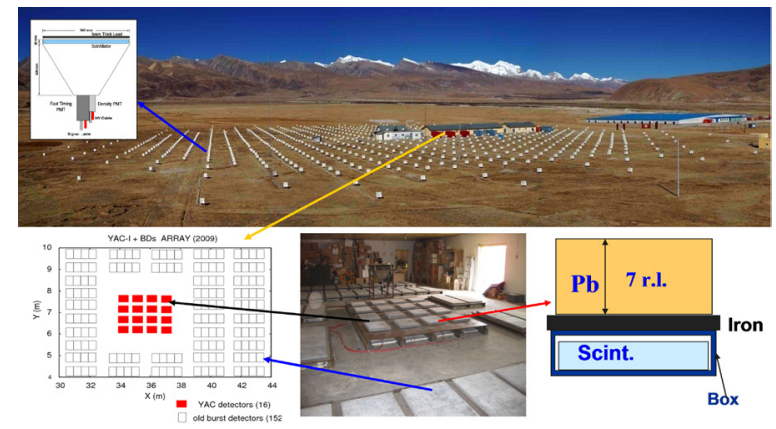

Figure 2. Schematic view of (YAC-I+Tibet-III) array. The TibetIII consists of 789 detector units, the YAC-I consists of 16 detector units.

in Tibet, China since May, 1st, 2009. YAC can work together with the Tibet-III array and a large muon detector as a hybrid experiment as shown in Fig.2. The YAC experiment is scheduled in three steps called YAC-I, YAC-II and YAC-III. YAC-I consists of 16 YAC detectors of size $40 \mathrm{~cm} \times 50 \mathrm{~cm}$, covering an area about $10 \mathrm{~m}^{2}$ as shown in Fig.2, which is used to check hadronic interaction models. YAC-II and YAC-III are used to obtain the individual component spectra of primary cosmic rays in a wide range over 3 decades between $50 \mathrm{TeV}$ and $100 \mathrm{PeV}$ in the near future.

The YAC-I detector has the same design as the YAC-II. The only difference between YAC-I and YAC-II is in spacing. Each YAC detector unit consists of lead plates of 3.5 $\mathrm{cm}$ (7 r.l.)(Fig.2) thick and a scintillation counter which detects the burst size induced by the high energy electromagnetic component in the air-shower core. The wide dynamic range between 1 MIP and $10^{6}$ MIPs is covered by 2 PMTs (Hamamatsu: R4125 and R5325) as shown in Fig.2. The response linearity of each YAC detector was calibrated by cosmic-ray single muons and by the accelerator beam of the BEPCII (Beijing Electron Positron Collider, IHEP, China).

The Tibet-III array consists of 789 detectors, covering an area of about $36900 \mathrm{~m}^{2}$. An event trigger signal is issued when any four-fold coincidence occurs in detectors recording more than 0.6 particles. The trigger rate is about $680 \mathrm{~Hz}$ and the dead time is $15 \%$. Tibet-III is used to measure the arrival direction $(\theta)$ and the air shower size $\left(N_{e}\right)$. The angular resolution is about 0.1 degree above $100 \mathrm{TeV}$ and the energy resolution is about $15 \%$ at $1 \mathrm{PeV}$ [1]. Online trigger condition for YAC is 'any 1' detector 'fired' (the discrimination threshold is about $30 \mathrm{mV}$ ).

If one YAC detector unit makes a trigger signal, all ADC data from all YAC units are recorded. Also the trigger signal is sent to the DAQ system for the Tibet-III array. ADC pedestal values are measured every 10 minutes. Each DAQ system has GPS clock module independently. The matching between YAC data and Tibet-III data is made using coincidence of GPS clocks and the trigger tag to the Tibet-III array.

\section{Simulation and Analysis}

We have carried out a detailed Monte Carlo ( MC ) simulation of air showers using the simulation code CORSIKA (version 7.5000) including EPOS-LHC, QGSJETII-04, SIBYLL2.1 and SIBYLL2.3 hadronic interaction models [10]. For the primary cosmic rays, we used three composition models, namely, "He-poor", "He-rich" and "H4a" models [1, 11, 12], The details of the assumed primary cosmic-ray flux are described in [13]. The minimum primary energy is set at $1 \mathrm{TeV}$. Primaries isotropically incident at the top of the atmosphere within the zenith angles from 0 to 60 degrees are injected into the atmosphere. The $\mathrm{MC}$ events are randomly dropped onto the detector array plane, $15 \mathrm{~m}$ wider on each side of the YAC-I array. The dropping area has been checked to be wide enough to contain $99.5 \%$ EAS events under our event selection conditions (see blow in the text).

All detector responses is based on the detector simulation code Geant4 (version 9.5) [14]. We confirmed that the shape of the energy loss distribution of YAC, which is determined by probe calibration simulation, shows a reasonable agreement with the charge distribution of the experimental data [13].

The simulated events are passed through the same analysis chains as the experimental data. Normally, the following parameters of (YAC-I+Tibet-III) are used to characterize an air-shower core events:

$N_{b}$ - the number of shower particles under the lead plate of a detector unit;

$N_{h i t}$ - the number of "fired" detector units with $N_{b} \geq$ a given threshold value;

$N_{b}{ }^{\text {top }}$ - the maximum burst size among fired detectors;

$\sum N_{b}$ - the total burst size of all fired detector units;

$<R>$ - the mean lateral spread, $<R>=\sum r_{i} /\left(N_{h i t}-1\right)$;

$<N_{b} R>$ - the mean energy-flow spread;

$<N_{b} R>=\sum\left(N_{b i} \times r_{i}\right) / N_{h i t}$, where $N_{b i}$ and $r_{i}$ are the burst size in the $i^{\text {th }}$ fired detector unit and the lateral distance from the air shower core to the center of the $i^{\text {th }}$ fired detector, respectively;

$N_{e}$ - the air shower size; it is estimated by fitting the lateral density distribution using the NKG function [1];

$\theta$ - the arrival direction of the air-shower.

In order to select the high-energy core events, we set $N_{b}{ }^{\text {top }} \geq 1500$ to reject events falling far from the array. The final data-selected condition is : $N_{b} \geq 200, N_{h i t} \geq 4$, $N_{b}{ }^{\text {top }} \geq 1500, N_{e} \geq 8000$. In this paper, we used the experimental data set obtained from May, 2009 through January, 2010. An event coincidence between AS events and YAC-I events is made by their arrival time. Deadtime corrections of $18 \%$ for AS trigger system and $15 \%$ for YAC-I trigger system are taken into account. The data sample coming from successful coincidence corresponds to a live time of 106.05 days. The statistics of such selected core events in MC simulation and experimental data as shown in Table1 The detection efficiency $\mathrm{S} \Omega A_{\text {eff }}$ is shown in Fig.3 


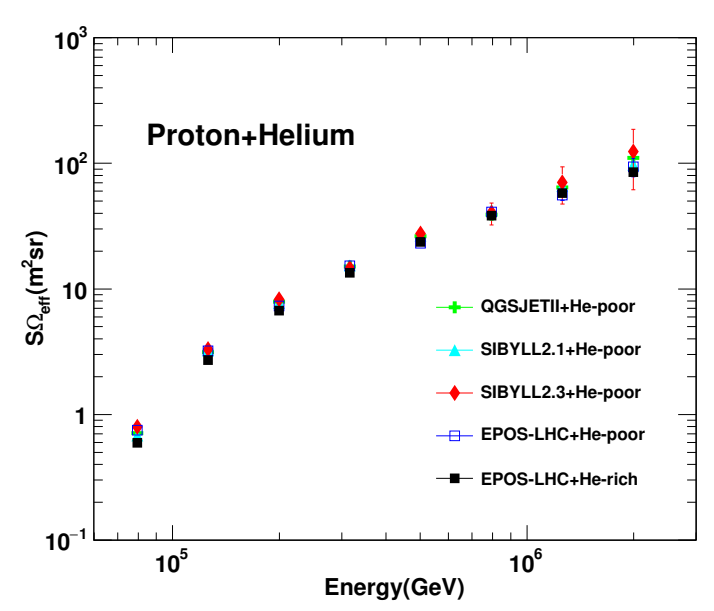

Figure 3. Efficiency $S \Omega$ of YAC-I array for Proton+Helium under the models mentioned above.

Table 1. Statistics of air-shower core events in MC simulation and experiment.

\begin{tabular}{c|c}
\hline MC Model & Selected core events \\
\hline EPOS-LHC+He-rich & 680989 \\
EPOS-LHC+He-poor & 21726 \\
QGSJETII-04+He-poor & 21856 \\
SIBYLL2.3+He-poor & 10152 \\
SIBYLL2.1+He-poor & 19176 \\
\hline Expt.data & $\mathbf{3 4 1 6}$ \\
\hline
\end{tabular}

\section{Check of interaction model and primary composition model dependence}

First, we checked the interaction model and primary composition model dependence by using (YAC1+Tibet-III) experimental data. The comparison of some observable parameters between YAC-I experimental data and MC simulation data calculated for various combination of interaction and primary composition models are shown in Fig.4 which shows that all interaction models produce no serious differences among their results. The experimental data are consistent with those MC distribution, which mean that there exists no detection bias.

\section{Primary $(\mathrm{P}+\mathrm{He})$ energy spectrum}

The selection of the (proton $+\mathrm{He}$ )-induced events is made using a feed-forward artificial neural network (ANN) [15], whose applicability to our experiment was well confirmed by the Monte Carlo simulation [4]. We examine four cases, (EPOS-LHC + He-poor), (QGSJETII-04 + He-poor), (SIBYLL2.1+He-poor) and (SIBYLL2.3+Hepoor) models. One MC data set was divided into two subsets, the one is used for training the ANN, and the other for estimating the ability of the ANN to classify the nuclear species. Then, the training MC data subset is fed to the ANN in a number of training cycles of 2000. To train
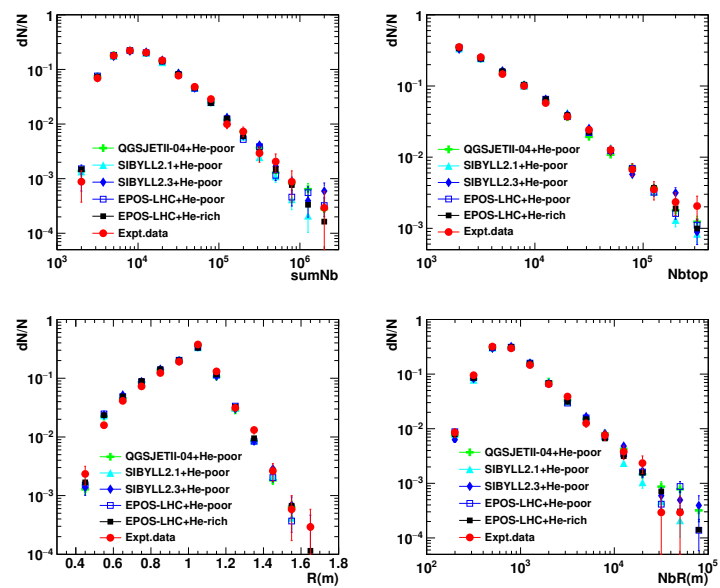

Figure 4. The comparison of some observable parameters $\left(\sum N_{b}\right.$, $\left.N_{b}{ }^{\text {top }},\langle R\rangle,\left\langle N_{b} R\right\rangle\right)$ between experimental data and MC simulation which are calculated for various combination of interaction and composition models.

Table 2. The average purity and efficiency of the selected events by $T_{c} \leq 0.4$

\begin{tabular}{c|cc}
\hline MC Model & Purity (\%) & Efficiency (\%) \\
\hline EPOS-LHC+He-poor & 93.5 & 80.4 \\
QGSJETII-04+He-poor & 93.7 & 83.0 \\
SIBYLL2.3+He-poor & 94.3 & 82.3 \\
SIBYLL2.1+He-poor & 93.0 & 80.0 \\
\hline
\end{tabular}

the ANN in separating (proton+He) from others, the input patterns for (proton+He) and other nuclei are set to 0 and 1, respectively. After the training, the other MC data subset is used to estimate the purity and the selection efficiency of (proton+He). Then, the ANN output pattern value $(\mathrm{T})$ is a real number from 0 to 1 . The $\mathrm{T}$ distributions in the (EPOS-LHC + He-poor) model is presented in Fig.5, together with the experimental data. One can see that the experimental data is in good agreement with the $\mathrm{MC}$ prediction, and that the (proton $+\mathrm{He}$ )-induced events are clearly separated from other nuclei. We define a critical value of $\mathrm{T}$ to separate (proton $+\mathrm{He}$ ) from others requiring the high purity, which reduces the effect of the contamination, and the high selection efficiency of the (proton $+\mathrm{He}$ ) events, which reduces the statistical error. Since these two factors are of a competing nature, the purity and the selection efficiency are calculated as a function of the critical value $T_{c}$ and its value is set as 0.4 where average purity and selection efficiency over the whole energy range are greater than approximately $95 \%$ and $76 \%$ for all models, respectively. It was confirmed that the final result does not depend on the different choice of the $T_{c}$ around 0.4 . The value of the average purity and efficiency are listed in Table 2 and their mutual deviation among different models being within $4 \%$ assures us the quality of the (proton $+\mathrm{He}$ ) selection. 


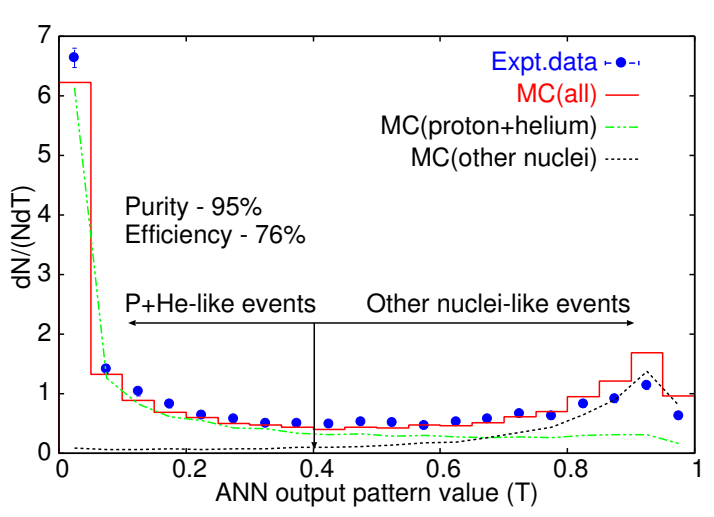

Figure 5. ANN output pattern value (T) distributions compared with MC ( EPOS-LHC + He-poor model ). The average purity and selection efficiency over whole energy range of $(\mathrm{P}+\mathrm{He})$ like events are $95 \%, 76 \%$ at $T_{c}=0.4$.

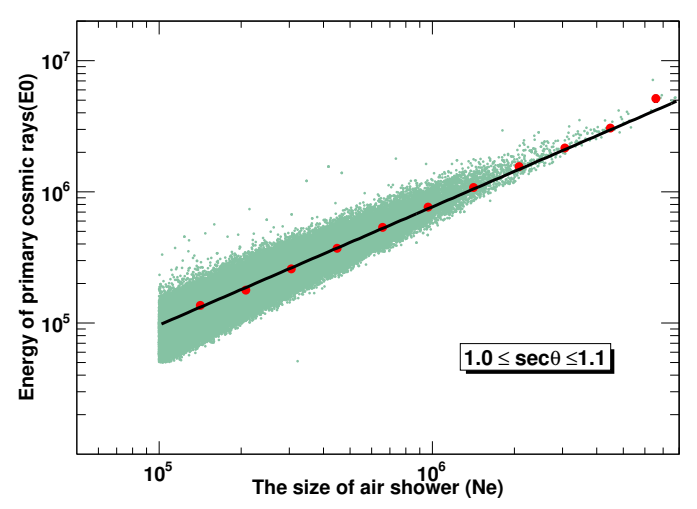

Figure 6. Scatter plots of the primary energy $E_{0}$ and the estimated shower size $N_{e}$ of $(\mathrm{P}+\mathrm{He})$-like events based on (EPOS$\mathrm{LHC}+\mathrm{He}$-poor) model with $\sec \theta \leq 1.1$.

The air-shower size in each event is estimated by fitting the lateral density distribution using a modified NKG function. The air-shower size resolution is about $5 \%$ around the primary energy of $1000 \mathrm{TeV}$ [1]. The primary energy of cosmic ray is calculated by the function $E_{0}=\mathrm{a} \times N_{e}{ }^{b}$, which is shown in Fig.6, and the energy resolution is about $25 \%$ at around $200 \mathrm{TeV}$.

Finally, we can obtain the primary $(\mathrm{P}+\mathrm{He})$ spectrum based on the newest model (EPOS-LHC+Herich), (EPOS-LHC+He-poor), (QGSJETII-04+He-poor), (SIBYLL2.1+He-poor) and (SIBYLL2.3+He-poor) together with the results from other experiments as shown in Fig. 7

As seen in Fig.7, we found :

1) The obtained $\mathrm{P}+\mathrm{He}$ spectrum is smoothly connected with direct observation data below $100 \mathrm{TeV}$ and also with our previously reported results at higher energies within statistical errors;

2) The knee of the $(\mathrm{P}+\mathrm{He})$ spectra is located around $400 \mathrm{TeV}$;

3) The interaction model dependence in deriving the primary $(\mathrm{P}+\mathrm{He})$ spectra is found to be small (less than

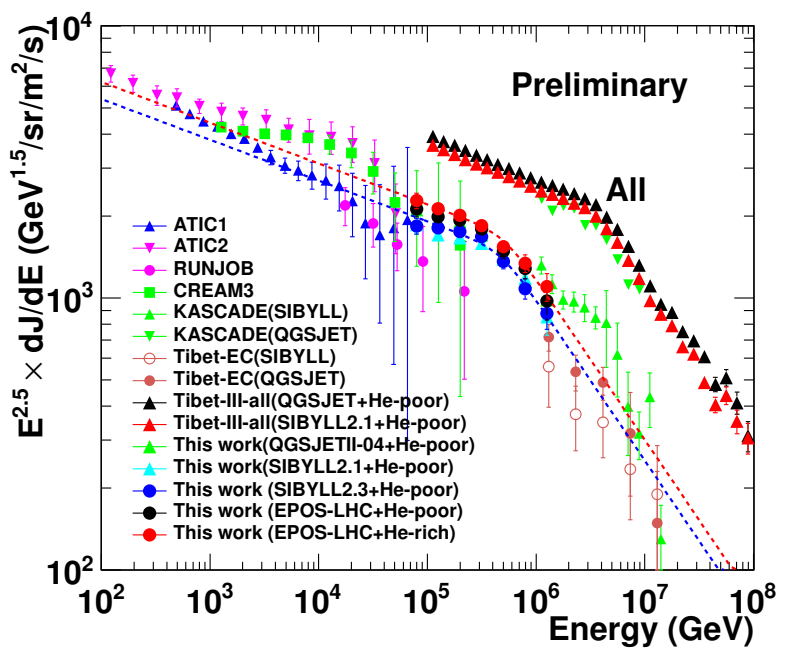

Figure 7. Differential energy spectra of Proton+Helium obtained by the present work compared with other experiments: ATIC1 [16], ATIC2 [17], RUNJOB [18], CREAM3 [19], KASCADE [5], Tibet-ECs [4].

$25 \%$ in absolute intensity, $10 \%$ in position of the knee), and the composition model dependence is less than $10 \%$ in absolute intensity.

\section{Summary}

YAC-I shows the ability and sensitivity in checking the hadronic interaction models. Based on the "Hepoor" primary model, we estimate that the difference of EPOS-LHC, QGSJETII-04, SIBYLL2.3 and SIBYLL2.1 is within $20 \%$ in our concerned energy region. High core events are very sensitive to the light components in CRs and the core parameters of $\sum N_{b}, N_{b}{ }^{t o p},\langle R\rangle$, and $\left\langle N_{b} R\right\rangle$ are very useful to separate the light components from all the observed events using an ANN technique. The flux of high energy core events is sensitive to the light components, and since the interaction model seems to depend strongly on the production of high energy core events, we can obtain the energy spectrum of light components in primary CRs with sufficient accuracy as discussed in the YAC-I experiment. (YAC1+Tibet-III ) could measure protons and heliums spectra above $50 \mathrm{TeV}$ which is shown to be smoothly connected with direct observation data at lower energies and also with our previously reported results at higher energies. We also found that the knee of the $(\mathrm{P}+\mathrm{He})$ spectra is located around $400 \mathrm{TeV}$. The interaction models dependence in deriving the $(\mathrm{P}+\mathrm{He})$ spectra are found to be small (less than $20 \%$ in absolute intensity, $10 \%$ in position of the knee ), and the composition model dependence is less than $10 \%$ in absolute intensity, and various systematic errors are under study now. The next phase experiment YACII will measure the primary energy spectrum of 4 mass groups of $\mathrm{P}, \mathrm{He}, 4<\mathrm{A}<40, \mathrm{~A}>40$ in the $5 \times 10^{13} \mathrm{eV}$ to $10^{16} \mathrm{eV}$ range covering the knee. 


\section{Acknowledgements}

The collaborative experiment of the Tibet Air Shower Arrays has been performed under the auspices of the Ministry of Science and Technology of China and the Ministry of Foreign Affairs of Japan. This work is supported by the National Key R\&D Program of China (No.2016YFE0125500), by the Grants from the National Natural Science Foundation of China (Nos.11533007,11673041,11603047,11275212 and 11803038), and by the Key Laboratory of Particle Astrophysics, Institute of High Energy Physics, CAS. This work is supported by the joint research program of the Institute for Cosmic Ray Research (ICRR), The University of Tokyo.

\section{References}

[1] M. Amenomori et al., Astrophys. J. 678 (2008) 1165.

[2] J. R. Hörandel, Astropart. Phys. 19 (2003) 193.

[3] J. R. Hörandel, Astropart. Phys. 21 (2004) 241.

[4] M. Amenomori et al., Phys. Lett. B 632 (2006) 58.

[5] T. Antoni et al., Astropart. Phys. 24 (2005) 1.

[6] J. P. Wefel et al., in Proc. 29th Int. Cosmic Ray Conf. (Pune) 3 (2005) 105.
[7] K. Asakimori et al., Astrophys. J. 502 (1998) 278.

[8] A. V. Apanasenko et al., Astropart. Phys. 16 (2001) 13.

[9] Y. S. Yoon, H. S. Ahn, T. Anderson, et al., in Proc. 31st Int. Cosmic Ray Conf. (Łódź) (2009).

[10] D.Heck et al., Forschungszentrum Karlsruhe Report FZKA 6019 (1998).

[11] M. Shibata, Y. Katayose, J. Huang and D. Chen, Astrophys. J. 716 (2010) 1076.

[12] T.K. Gaisser, Astroparticle Physics 35, 801 (2012).

[13] L.M. Zhai, J. Huang, D. Chen, et al, Journal of Physics G: Nuclear and Particle Physics 42, 045201 (2015)

[14] S. Agostinelli, et al., NIM, A 506:250-303,(2003).

[15] L. Lonnblad, et al., Comp. Phys. Comm., 81: 185220, (1994).

[16] Ahn, H. S., et al., in Proc. 28th ICRC (Tsukuba), 4: 1833-1836, (2003).

[17] Wefel, J. P., et al., in Proc. 29th ICRC (Pune), 3: 105108, (2005).

[18] Apanasenko, A. V., et al., Astropart. Phys., 16: 1346, (2001).

[19] Y.S. Yoon, T. Anderson et al., ApJ, 839:5, (2017). 